\title{
BMJ Open Identifying patient-important outcomes for treatment of bipolar disorder: a systematic review protocol
}

\author{
Alessia D'Elia (D) , ${ }^{1,2}$ Olivia Orsini, ${ }^{3}$ Stephanie Sanger, ${ }^{4}$ Alannah Hillmer (D) ,,2 \\ Nitika Sanger (D) ,,5 Balpreet Panesar (D) ,1,2 Myanca Rodrigues (D) , 1,6 \\ Flavio Kapczinski, ${ }^{1}$ Lehana Thabane (D) , ${ }^{7,8,9}$ Zainab Samaan (1) ${ }^{1,10}$
}

To cite: D'Elia A, Orsini 0, Sanger S, et al. Identifying patient-important outcomes for treatment of bipolar disorder: a systematic review protocol. BMJ Open 2021;11:e050453. doi:10.1136/ bmjopen-2021-050453

- Prepublication history for this paper is available online. To view these files, please visit the journal online (http://dx.doi org/10.1136/bmjopen-2021050453).

Received 09 March 2021 Accepted 06 December 2021

Check for updates

(c) Author(s) (or their employer(s)) 2021. Re-use permitted under CC BY-NC. No commercial re-use. See rights and permissions. Published by BMJ.

For numbered affiliations see end of article.

Correspondence to

Dr Zainab Samaan;

samaanz@mcmaster.ca

\section{ABSTRACT}

Introduction Treatment of bipolar disorder is the focus of several clinical trials, however the understanding of the outcomes for establishing treatment effectiveness within these trials is limited. Further, there is limited literature which reports on the outcomes considered to be important to patients, indicating that patient perspectives are often not considered when selecting outcomes of effectiveness within trials. This protocol describes a systematic review which aims to describe the outcomes being used within trials to measure treatment effectiveness, commenting on the inclusion of patient-important outcomes within previous trials.

Methods and analysis This protocol is reported using the Preferred Reporting Items for Systematic Reviews and Meta-analyses Protocols statement. OVID MEDLINE, OVID Embase, OVID APA PsycINF0, Web of Science, the Wiley Cochrane Library, ClinicalTrials.gov and the International Clinical Trials Registry Platform databases will be searched for eligible studies. Screening, full-text and data extraction stages will be completed in duplicate using the Covidence platform for systematic reviews. Eligible studies will include clinical trials of interventions in bipolar disorder, in order to identify outcomes used to assess treatment effectiveness, and qualitative studies, to determine which outcomes have been reported as important by patients. Risk of bias for included studies will be assessed using the Cochrane Risk of Bias Tool for randomised controlled trials, and the Newcastle-Ottawa Scale for observational research.

Ethics and dissemination This review will involve dissemination to key stakeholders, including primary end users such as patients, clinicians and trialists. Knowledge translation tools will be generated to share the relevant conclusions of this review. Results will be communicated to the scientific community through peer-reviewed publications, conferences and workshops. No ethics approval will be sought as this study is based on literature. PROSPERO registration number CRD42021214435.

\section{INTRODUCTION}

Bipolar disorder type 1 (BD-I) is a chronic mood disorder associated with severe depressive and manic episodes. ${ }^{1}$ Though among the top eight most prevalent conditions worldwide, ${ }^{2} \mathrm{BD}-\mathrm{I}$ is very difficult to diagnose

\section{Strengths and limitations of this study}

- The proposed review employs a two-pronged approach to appraise outcomes used by trialists to assess treatment effectiveness in clinical trials of bipolar disorder I and describe patient-important outcomes.

- Strong methodological design developed in accordance with the Preferred Reporting Items for Systematic Reviews and Meta-analyses Protocol guidelines for transparent reporting.

- The planned analysis for reporting trials outcomes includes stratification of outcomes according to population, intervention type and mood state.

- Later analysis, including ability to conduct thematic analysis, may be impacted by large variability in the types of outcomes being used in trials of bipolar disorder I.

- Restriction to include studies published in English may lead to language bias.

as a result of heterogeneous illness presentation which often leads to misdiagnosis as major depressive disorder. ${ }^{3}$ Wide variability in symptom presentation, often impacted by age of diagnosis and other sociodemographic factors, ${ }^{5-7}$ presents challenges not only to diagnosis, but also to the selection of an appropriate course of treatment. ${ }^{8}$ BD-I has significant impact on patients' lives including recurrence of psychiatric symptoms, ${ }^{9}$ comorbid psychiatric and medical disorders, ${ }^{10}{ }^{11}$ loss of function, ${ }^{12}{ }^{13}$ increased risk of mortality, ${ }^{14}{ }^{15}$ poor quality of life,${ }^{16} 17$ cognitive difficulties ${ }^{18}$ among others. These pervasive and diverse impacts highlight the importance of determining which treatments most effectively manage specific outcomes, and for whom specific outcomes are of particular concern.

Current treatment of BD-I is medication paired with adjunct psychotherapy and is typically indicated by whether depressive or manic symptoms are more dominant within 
a particular individual. Though medications can be effective in managing mood symptoms, these symptoms and others, such as cognitive and metabolic changes, are often persistent and require adjunct psychotherapy and other interventions. ${ }^{19}$ Indeed, current guidelines recommend the combination of psychotherapy and medication in order to obtain successful symptom management and remission. ${ }^{19}$ Several reviews and guidelines have been published which provide evidence for such treatments, however, there is little consensus on which interventions are most effective and for what outcomes.

Further, little research investigates which treatment outcomes are most important to patients with BD-I. There is great variety among trials in the outcomes selected to indicate treatment success, with some studies considering treatments to be effective if patients achieve reductions in number of episodes or hospitalisations, ${ }^{20}$ some looking for reductions in mood symptom severity or burden, ${ }^{21}$ and others using measures such as the number of days without mood symptoms as an indicator of success. ${ }^{22}$ Even in trials which measure effectiveness using the same type of outcome or instrument, time points, thresholds and definitions of effectiveness can differ greatly. While variability in outcomes is expected, this raises questions on whether these outcomes, and the way they are measured, reflect patient perspectives or can appropriately approximate the outcomes considered to be important to patients.

Some research has identified the weight or relative importance of existing outcomes often examined in trials, such as depressive and manic symptoms, social functioning, and quality of life, in addition to collecting important outcomes through focus groups. ${ }^{23}$ However, such studies are often small convenience samples, potentially failing to reflect the full range of outcomes deemed important by patients, or represent the perspectives of patients at varied phases of the disorder, such as those in active and acute phases. ${ }^{23}$ The exclusion of patients' specific perspectives on what they need out of treatment for bipolar disorder emphasizes the need to evaluate the extent to which trial outcomes are in agreement with patient perspectives. Further, it is essential that this assessment is disorder-specific, given that experiences of BD-I are distinct and relevant outcomes may differ importantly from those of other similar disorders. In order to do so, it is essential to not only appraise the available literature related to outcome measurements, but also the literature pertaining to patient-important outcomes (PIO) in BD-I.

\section{Rationale}

Reviews which aim to systematically examine and describe the outcomes used within clinical trials of treatments for BD-I to establish treatment effectiveness are needed. Given that BD-I is distinct from other bipolar spectrum disorders, it is essential to conduct this review within BD-I patients specifically. Such reviews are essential in order to facilitate an understanding of the inclusion of PIOs in trials of BD-I. Therefore, it is important to examine the level of agreement between trialists, and moreover between trialists and patients, in order to determine whether measures of effectiveness truly reflect the needs of patient populations. This review will aim to appraise the outcomes used as a means to support future investigation of the need for a core outcome set for trials of BD-I.

\section{Objectives}

The purpose of this systematic review is to investigate the outcomes used to measure treatment effectiveness within trials for treatment of BD-I. Specifically, the aims of this review are:

1. Summarise the outcomes (clinical scales, biological or social markers, etc) used within clinical trials to measure treatment effectiveness, and report how these outcomes are assessed.

2. Review the observational and qualitative research related to PIO for bipolar disorder (ie, goals and markers of treatment success identified as important by patients).

\section{METHODS AND ANALYSIS}

We will conduct a systematic review of outcomes reported in published trials and observational/qualitative research. The reporting of this protocol reflects the guidelines of the Preferred Reporting Items for Systematic Reviews and Meta-analyses (PRISMA) Protocols statement. ${ }^{24}$

Two search strategies will be used in order to investigate our two objectives of interest. The specific inclusion and exclusion criteria are described for each objective. Further, the population, intervention, comparators, outcomes, timing and setting for studies meeting inclusion are specifically outlined.

\section{Eligibility criteria for objective 1}

The inclusion criteria for objective 1 is randomised controlled trials (RCTs) testing the effectiveness of treatment interventions in BD-I that report intervention outcomes.

\section{Participant population}

Trials testing treatments within (1) patients with BD-I and (2) a mixed sample of participants BD-I and II, will meet inclusion. While objective 1 endeavours to report on outcomes used in trials of BD-I specifically, trials with mixed BD-I and II samples will be included since a large proportion of trials evaluating treatment for BD-I involve mixed samples, and exclusion of these studies would preclude rigorous appraisal of trial outcomes.

\section{Intervention}

Trials investigating psychotherapy and pharmacotherapy interventions will meet inclusion. Psychotherapies included in this review will include any psychotherapeutic intervention specifically intended to treat bipolar disorder, such as cognitive-behavioural therapy (CBT), behavioural activation (BA), interpersonal psychotherapy, interpersonal and social rhythm therapy (IPSRT), familyfocused therapy (FFT) and psychoeducation. Psychotherapy interventions were selected for inclusion based 
on their classification as a (1) form of psychotherapy and (2) previous evidence of effectiveness in treatment of bipolar disorder. Pharmacotherapeutic interventions will include medications being tested for effectiveness in treating symptoms of bipolar disorder. Trials meeting inclusion will include an intervention, a comparator and at least one outcome measure or end point. Trials which include both group and individual-based interventions will be eligible for this review. Interventions which include combination therapies (ie, medication and a form of psychotherapy) will be included.

\section{Comparators}

No restrictions will be placed on comparators.

\section{Outcomes}

All outcomes pertaining to effectiveness of interventions will be included (ie, clinical scales, biological or social markers).

\section{Studies}

RCTs will be included with no restrictions on study setting (ie, clinic or community-based settings). No restrictions on age, country, income status or type of recruitment will be applied. Only complete studies in humans and written in the English language will be eligible. In addition to the identified databases, clinical trial registries will be searched; in the case of registration numbers with multiple associated publications, the most recent publication will be selected for inclusion if not already identified in the search.

\section{Time}

No restrictions on time will be applied.

\section{Exclusion criteria}

Animal studies, preliminary reports, pilot/feasibility studies, abstracts, presentations, interim-analyses and trials which investigate interventions other than those outlined above will be excluded. Interventions designed to address challenges within families, or couples that are explicitly not intended to treat bipolar disorder will not be included. Trials testing the effects of discontinuing treatment will not be eligible for inclusion. Studies investigating pharmacotherapeutics and psychotherapeutics not intended for treating bipolar disorder (ie, treating obesity or medication-related side effects), will be excluded.

\section{Eligibility criteria for objective 2}

Participant population

Studies with a BD-I population.

\section{Outcomes}

PIO (ie, any outcome, marker or measure) reported by participants by BD-I.

\section{Studies}

The inclusion criteria for objective 2 are observational and qualitative studies. Qualitative studies meeting the inclusion criteria will be those involving focus groups and interviews to determine what outcomes are reported by patients with BD-I to be important for measuring treatment success. There will be no restrictions on age, country, income status, type of recruitment or study setting.

\section{Time}

There will be no restrictions based on time.

\section{Exclusion criteria}

The exclusion criteria for objective 2 are studies for which the aims are unrelated to identifying PIO, and studies whose population is not patients with BD-I.

\section{Outcomes and prioritisation}

The first objective of this systematic review will be to report the outcomes used to measure the effectiveness of the treatment being tested. These outcomes will be extracted from RCTs, and may include depressive or manic symptoms, quality of life measurements, or outcomes related to social adversity, such as employment. Given that the population of interest for this review is patients with BD-I, outcomes explored within these patients will qualify for extraction. Therefore, extracted outcomes will be stratified based on population, with outcomes in studies of BDI only being reported separately from outcomes in studies with mixed BDI and II samples.

The second objective will be to examine the literature pertaining to PIO for BD-I. Observational and qualitative studies will be examined to determine which outcomes are reported by patients to be important measures of treatment success.

\section{Information sources}

The selection of databases and the corresponding search strategies were developed through partnership with a Clinical Services Librarian from the Health Sciences Library at McMaster University. Eligible studies will be identified through searches of the following databases: OVID MEDLINE (1946-Current), OVID Embase (1974-Current), OVID APA PsycINFO (1987-Current), Web of Science (1976-Current), the Wiley Cochrane Library (1999-Current), ClinicalTrials.gov and the International Clinical Trials Registry Platform. Databases will be searched for all sources of literature, including grey literature, from inception to the date of search, which will be reported in the final systematic review. The search strategy for each objective for one database is described in table 1 .

\section{Data management}

Articles identified through the search strategy will be imported to Zotero, and then the Covidence platform. ${ }^{25}$ Title and abstract, full-text and data extraction phases will be managed through this platform. Members of the research team who have not used Covidence before will be trained through online tutorials and an additional training session will be 


\begin{tabular}{|c|c|}
\hline Database & Search strategy \\
\hline OVID MEDLINE & 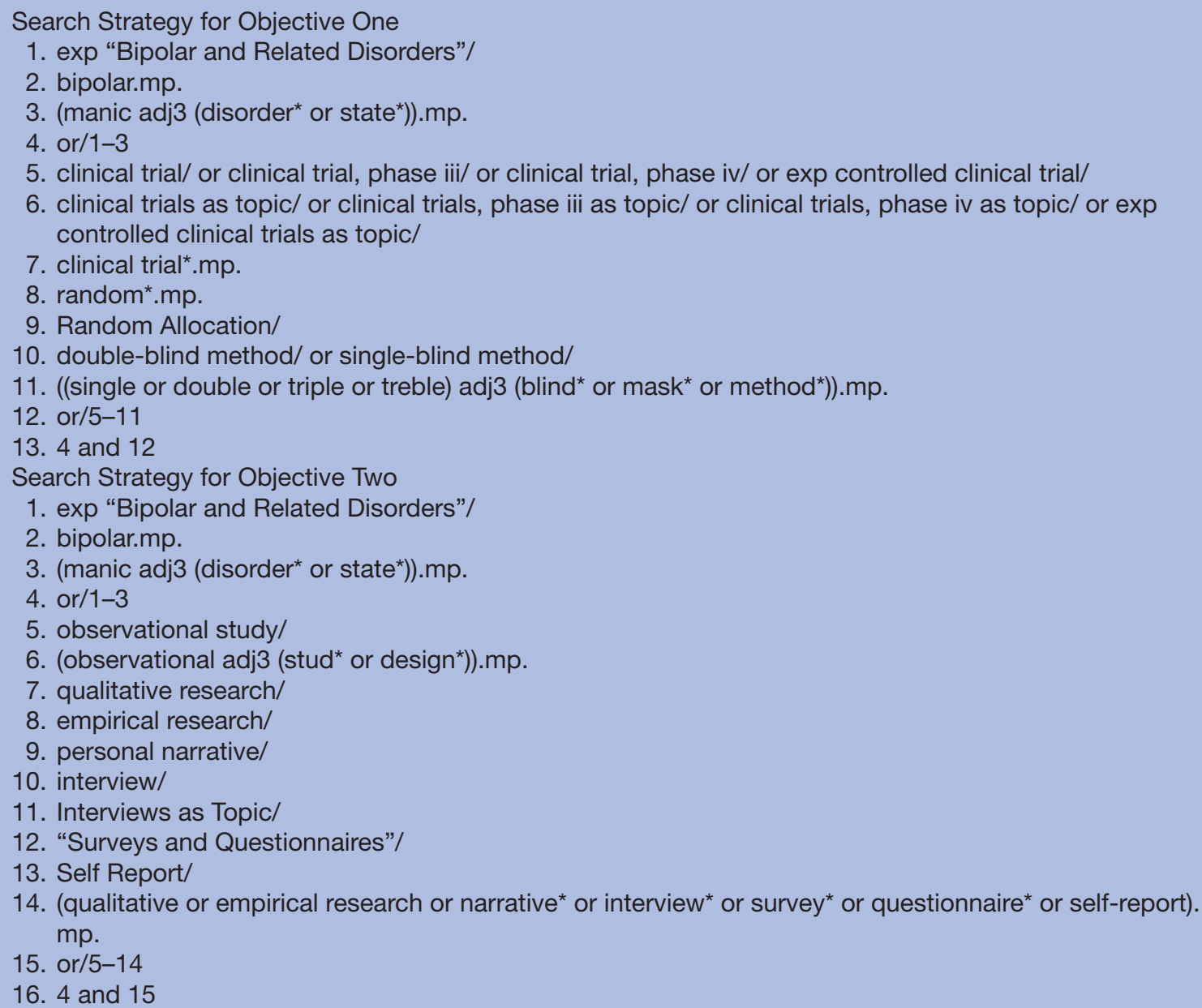 \\
\hline
\end{tabular}

conducted with all reviewers to ensure familiarity and consistency of use at all stages. A calibration phase will be completed where reviewers will be asked to screen 25 articles on the platform, and responses will be reviewed to ensure understanding of the protocol and criteria.

\section{Selection process}

Each citation identified will be reviewed by two reviewers independently at title and abstract and then full-text stages, using the eligibility criteria described above. Those citations meeting eligibility during these phases will be included for data extraction. Disagreements between reviewers will be resolved by another reviewer to reach consensus. Level of agreement between reviewers will be assessed and the kappa statistic will be reported.

A flow diagram (figure 1) summarising the screening of all studies will be included in the final review. Studies included in the data extraction phase will be described in a table, which will be structured in keeping with the guidelines specified by the PRISMA guidelines. ${ }^{26}$ Reasons for exclusion or inclusion will be reported.

\section{Data collection process}

Data extraction forms will be built on Covidence and completed blindly, in duplicate. Separate data extraction forms will be constructed for objective 1 and 2, and the forms will be pilot tested by all reviewers to ensure the quality of the extraction and the comprehensiveness of the forms. The data collection forms will include the following items: author, year, country, title of journal, number of participants, name of intervention, diagnosis, diagnostic criteria, mood state, phase of disorder, inclusion criteria, exclusion criteria, type of population (ie, inpatient, outpatient or community), ethnicity, cultural factors, mean age, details on special populations (low income, pregnant, veteran, etc) and study design. Where data are missing, authors will be contacted, and all correspondence will be noted.

For objective 1, the data collection form will also include details on form of treatment (ie, CBT, BA, 


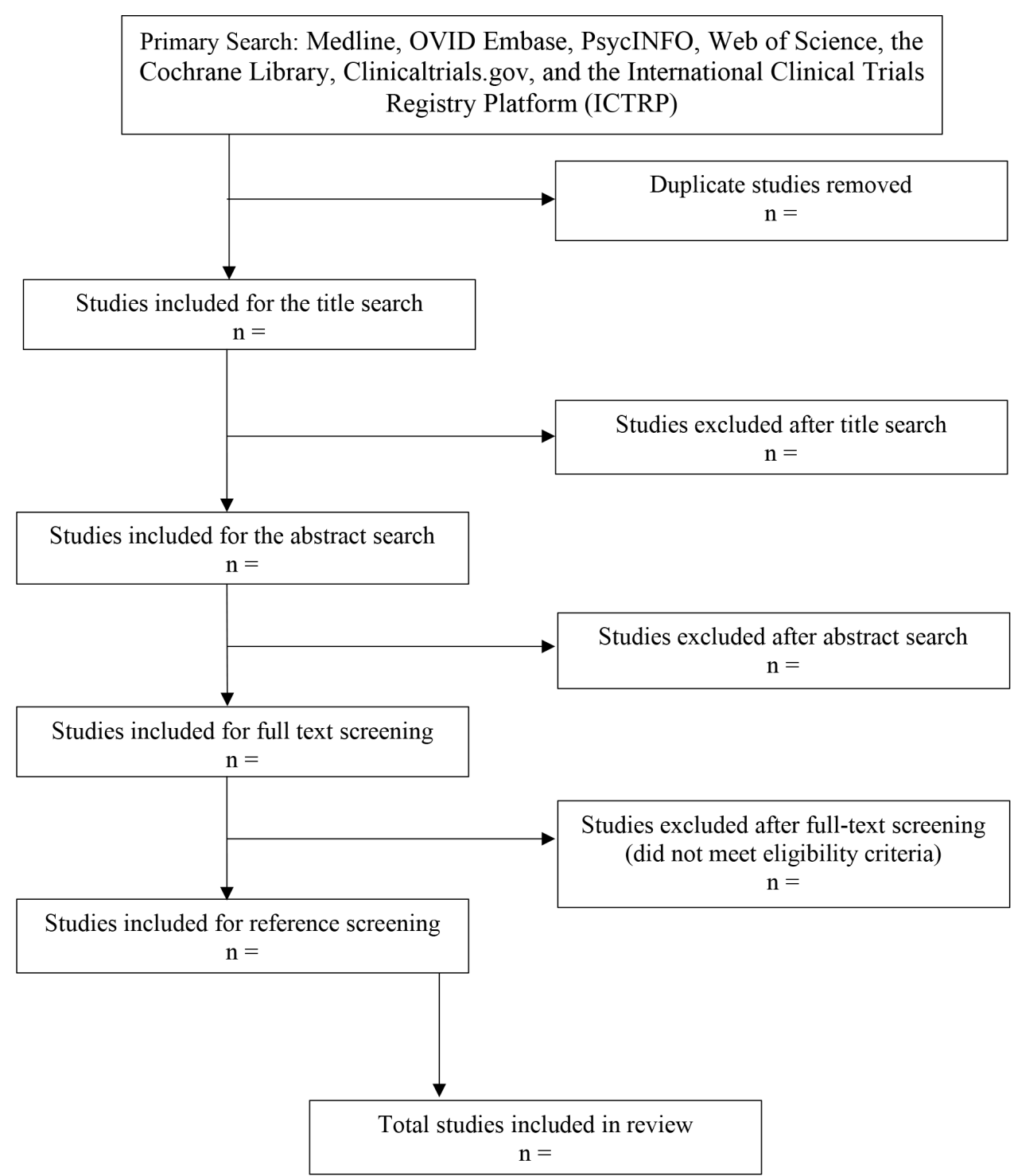

Figure 1 Flow diagram of included studies. The following flow diagram will be used in the final review to describe each phase of review, and the final number of included studies.

etc). Study outcomes used to assess effectiveness in each study will be recorded, and the following information about these outcomes will be extracted: the type of outcome, the definition of the outcome, how often it is measured, and how it is measured. For objective 2, the following additional information will be extracted from the observational and qualitative literature: the outcomes reported by patients to be important as markers of treatment success and the themes of PIO reported.

The anticipated date for data collection for objective 1 is December 2021, and objective 2 is February 2022.

\section{Risk of bias assessment}

In duplicate, individual studies will be examined to assess the quality of the included studies. For trials assessed through objective 1, the Cochrane Risk of Bias Tool $^{27}$ will be used. The standard cut offs reported for this tool in the literature is a score of 6 or higher; studies meeting this cut-off will be included in subgroup analysis. For studies with an observational or qualitative design in objective 2, the NewcastleOttawa Scale ${ }^{28}$ will be used. Studies scoring 5 or lower on this tool will be included in subgroup analysis.

\section{Data synthesis}

The outcomes extracted for the first objective of this systematic review will be qualitatively reported. Descriptions (type of outcome, definition, method and timing of outcome data collection) of outcomes will be provided for each eligible study. Reporting of outcomes will also be stratified by population (BD-I, or BD-I and II mixed populations), then by type of intervention (psychotherapy, pharmacotherapy or a combination) and by mood state, for studies where this information is provided. The rationality for the selection of outcomes will be summarised for each study. 
For the second research objective, thematic analysis will be conducted to group outcomes reported by patients to be important. Thematic analysis will be sensitive to the types of outcomes reported by patients, and therefore, grouping will be selected based on the themes that appear. Results of qualitative and observational studies will be summarised.

Within both objectives, stratification according to sociodemographic and clinical variables will be conducted to explore the relevance of these characteristics to PIO. For objective 1, reported outcomes will be stratified by sociodemographic characteristics including ethnicity, age, sex and gender, and clinical characteristics like phase of disorder, disorder onset and additional treatment, where data is sufficient and available. For objective 2, analysis of PIO based on sociodemographic details such as ethnicity, age, sex and gender can be explored to identify differences in outcomes based on important identity factors. A qualitative summary of differences in outcomes reported will be presented. Where sufficient clinical data is available related to phase of disorder, disorder onset, and additional treatment, differences in PIO can be explored.

\section{Implications}

Through objectives one and two, this review aims to systematically appraise the literature in order to determine the current outcomes being used within trials, how these outcomes were measured and to whom they apply to establish treatment success. Through this study, we will draw conclusions on what outcome and endpoints exist within trials, determine how these outcomes were measured, and examine the extent to which PIO are included. Guided by the review findings, insight on relevant factors (mood state, phase of disorder, social factors, etc) will precipitate a more precise approach for the possible development of a core outcome set for BD-I. Planned stratification by intervention type, phase of disorder, and other important social factors will contribute to an understanding of the specific types of outcomes used for establishing the effectiveness of certain interventions, and uncover which outcomes are important given relevant social factors. The focus on the BD-I population will precipitate appropriate comparison of the outcomes of effectiveness used in trials to those reported as important by patients, in keeping with a precision-medicine approach.

\section{Limitations}

The planned review has limitations. The first objective of this review will include only a subset of the possible medications or therapies that have been previously tested in patients with BD-I, meaning that trials of medications or treatment for conditions or symptoms distinct from their BD-I diagnosis (ie, drugs targeting obesity, medication-related side effects) will not be included. This review will be limited to studies in English, which may lead to language bias.

\section{Patient and public involvement}

There was no patient or public involvement in the conception of this systematic review protocol.

\section{ETHICS AND DISSEMINATION}

The findings of this systematic review will be disseminated with important stakeholders and relevant communities. This review will provide a systematic appraisal of the outcomes used in trials to demonstrate effectiveness, as well as those that are reported by patients to be important. Through ongoing collaborations and partnerships with tertiary care centres, we aim to circulate findings to clinicians and patients. Tools such as summary reports and guidelines will be constructed in order to translate the results of this study to these primary end users. Findings will also be shared with researchers, knowledge users, learners and clinicians through conference presentations, workshops and scientific publications.

\section{Author affiliations}

${ }^{1}$ Psychiatry and Behavioural Neurosciences, McMaster University, Hamilton, Ontario, Canada

${ }^{2}$ Neuroscience Graduate Program, McMaster University, Hamilton, Ontario, Canada

${ }^{3}$ Department of Health Studies and Department of Psychology, University of Toronto Scarborough, Toronto, Ontario, Canada

${ }^{4}$ McMaster University Library, Hamilton, Ontario, Canada

${ }^{5}$ Medical Science Graduate Program, McMaster University, Hamilton, Ontario, Canada

${ }^{6}$ Health Research Methodology, McMaster University, Hamilton, Ontario, Canada ${ }^{7}$ Department of Health Research Method, Evidence, and Impact, McMaster University, Hamilton, Ontario, Canada

${ }^{8}$ Population Health Research Institute, McMaster University, Hamilton, Ontario, Canada

${ }^{9}$ Father Sean 0'Sullivan Research Centre, St. Joseph's Healthcare Hamilton, Hamilton, Ontario, Canada

${ }^{10}$ Faculty of Health Sciences, McMaster University, Hamilton, Ontario, Canada

\section{Twitter Myanca Rodrigues @MyancaRodrigues}

Contributors AD'E: contributed to the conception and design of the study and study protocol, the writing and final review of the manuscript, and developed search strategy and the data collection tool within Covidence. 00: contributed to the writing and final review of the manuscript. SS: contributed critically to the development of the search strategy and final review of the manuscript. AH, NS, BP, MR, FK and LT: provided critical revision and review of the final manuscript. ZS: contributed to the conception and design of the study, provided critical revision and provided approval of the final manuscript. All authors read and approved the final manuscript.

Funding This work is not funded by a specific grant. ZS received funding from Alternate Funding Plan (No. 20-10178-480925-75153), and Canadian Institutes for Health Research (Award No. PJT-156306).

Competing interests None declared.

Patient and public involvement Patients and/or the public were not involved in the design, or conduct, or reporting, or dissemination plans of this research.

Patient consent for publication Not applicable.

Provenance and peer review Not commissioned; externally peer reviewed.

Open access This is an open access article distributed in accordance with the Creative Commons Attribution Non Commercial (CC BY-NC 4.0) license, which permits others to distribute, remix, adapt, build upon this work non-commercially, and license their derivative works on different terms, provided the original work is 
properly cited, appropriate credit is given, any changes made indicated, and the use is non-commercial. See: http://creativecommons.org/licenses/by-nc/4.0/.

\section{ORCID iDs}

Alessia D'Elia http://orcid.org/0000-0002-6512-4704

Alannah Hillmer http://orcid.org/0000-0002-5535-7432

Nitika Sanger http://orcid.org/0000-0002-5883-1873

Balpreet Panesar http://orcid.org/0000-0002-5492-3615

Myanca Rodrigues http://orcid.org/0000-0001-7953-773X

Lehana Thabane http://orcid.org/0000-0003-0355-9734

Zainab Samaan http://orcid.org/0000-0002-5974-9361

\section{REFERENCES}

1 Phillips ML, Kupfer DJ, WHO. Bipolar Disorder 2 - Bipolar disorder diagnosis: Challenges and future directions. Lancet 2013.

2 WHO. The global burden of disease. World Health Organization, 2004.

3 Singh T, Rajput M. Misdiagnosis of bipolar disorder. Psychiatry 2006:3:57-63.

4 Lish JD, Dime-Meenan S, Whybrow PC, et al. The National depressive and manic-depressive association (DMDA) survey of bipolar members. J Affect Disord 1994;31:281-94.

5 Rowland TA, Marwaha S. Epidemiology and risk factors for bipolar disorder. Ther Adv Psychopharmacol 2018;8:251-69.

6 Leboyer M, Henry C, Paillere-Martinot M-L. Age at onset in bipolar affective disorders: a review. Bipolar Disord 2005;7:111-8.

7 Tsuchiya KJ, Byrne M, Mortensen PB. Risk factors in relation to an emergence of bipolar disorder: a systematic review. Bipolar Disord 2003:5:231-42.

8 Merikangas KR, Jin R, He J-P, et al. Prevalence and correlates of bipolar spectrum disorder in the world mental health survey initiative. Arch Gen Psychiatry 2011;68:241.

9 Vázquez GH, Holtzman JN, Lolich M, et al. Recurrence rates in bipolar disorder: systematic comparison of long-term prospective, naturalistic studies versus randomized controlled trials. Eur Neuropsychopharmacol 2015;25:1501-12.

10 Grande I, Berk M, Birmaher B. Bipolar disorder. Lancet 2016.

11 Steel Z, Marnane C, Iranpour C, et al. The global prevalence of common mental disorders: a systematic review and meta-analysis 1980-2013. Int J Epidemiol 2014;43:476-93.

12 Bowden CL. Bipolar disorder and work loss. Am J Manag Care 2005;11:S91-4.

13 Michalak EE, Yatham LN, Maxwell V. The impact of bipolar disorder upon work functioning: a qualitative analysis. Bipolar Disord 2007;9:126-43.
14 Crump C, Sundquist K, Winkleby MA, et al. Comorbidities and mortality in bipolar disorder: a Swedish national cohort study. JAMA Psychiatry 2013;70:931-9.

15 Almeida OP, McCaul K, Hankey GJ, et al. Risk of dementia and death in community-dwelling older men with bipolar disorder. $\mathrm{Br} \mathrm{J}$ Psychiatry 2016;209:121-6.

16 Pascual-Sánchez A, Jenaro C, Montes-Rodríguez JM. Quality of life in euthymic bipolar patients: a systematic review and meta-analysis. $J$ Affect Disord 2019;255:105-15.

17 IsHak WW, Brown K, Aye SS, et al. Health-related quality of life in bipolar disorder. Bipolar Disord 2012;14:6-18.

18 Martino DJ, Samamé C, Ibañez A, et al. Neurocognitive functioning in the premorbid stage and in the first episode of bipolar disorder: a systematic review. Psychiatry Res 2015;226:23-30.

19 Yatham LN, Kennedy SH, Parikh SV, et al. Canadian network for mood and anxiety treatments (CANMAT) and International Society for bipolar disorders (ISBD) 2018 guidelines for the management of patients with bipolar disorder. Bipolar Disord 2018;20:97-170.

20 Lam DH, Watkins ER, Hayward P, et al. A randomized controlled study of cognitive therapy for relapse prevention for bipolar affective disorder: outcome of the first year. Arch Gen Psychiatry 2003;60:145-52.

21 Parikh SV, Zaretsky A, Beaulieu S, et al. A randomized controlled trial of psychoeducation or cognitive-behavioral therapy in bipolar disorder: a Canadian Network for Mood and Anxiety treatments (CANMAT) study [CME]. J Clin Psychiatry 2012;73:803-10.

22 Zaretsky A, Lancee W, Miller C, et al. Is cognitive-behavioural therapy more effective than psychoeducation in bipolar disorder? Can $\mathrm{J}$ Psychiatry 2008;53:441-8.

$23 \varnothing \mathrm{E}$, Nylenna M, Nytrøen K. Patient-Important outcomes in the long-term treatment of bipolar disorder: a mixed-methods approach investigating relative preferences and a proposed taxonomy. Patient 2016.

24 Moher D, Shamseer L, Clarke M. Preferred reporting items for systematic review and meta-analysis protocols (PRISMA-P) 2015 statement. Rev Esp Nutr Humana y Diet 2016.

25 Innovation VH. Covidence systematic review software. Melbourne, Australia: Veritas Health Innovation, 2020. www.covidence.org. Melbourne Australia

26 Moher D, Liberati A, Tetzlaff J, et al. Preferred reporting items for systematic reviews and meta-analyses: the PRISMA statement. BMJ 2009;339:b2535.

27 Higgins JPT, Altman DG, Gøtzsche PC, et al. The Cochrane collaboration's tool for assessing risk of bias in randomised trials. BMJ 2011;343:d5928.

28 Wells GA, Shea B, O'connell D. The Newcastle-Ottawa scale (NOS) for assessing the quality if nonrandomized studies in meta-analyses, 2009. Available: http//www ohri ca/programs/clinical_epidemiology/ oxford htm [Accessed 19 Oct 2009]. 